\title{
Synthesis of (S)-omeprazole catalyzed by soybean pod peroxidase in water-in-oil microemulsions: optimization and modeling
}

\author{
yuanyuan Zhang ${ }^{1}$, Hui Tang ${ }^{1}$, Yashan Deng ${ }^{1}$, Depeng Li $^{1}$, Zhiyong Wang ${ }^{1}$, Huiling Li $^{1}$, \\ Xin Xin $\mathrm{Gao}^{1}$, and Fanye Wang ${ }^{1}$ \\ ${ }^{1}$ Qingdao University of Science and Technology
}

October 6, 2020

\begin{abstract}
Response surface methodology (RSM) was used to optimize the oxidation of the omeprazole sulfide to (S)-omeprazole catalyzed by environmentally friendly catalyst soybean pod peroxidase (SPP) in cetyltrimethylammonium bromide (CTAB)/isooctane/nbutyl alcohol/water water-in-oil microemulsions. With the initial concentration of SPP of $3200 \mathrm{U}$ ml-1, the conversion of the omeprazole sulfide, the (S)-omeprazole yield and ee were $93.75 \%, 91.56 \%$ and $96.08 \%$, respectively, under the optimal conditions: Wo of 15.85 , the concentration of $\mathrm{H} 2 \mathrm{O} 2$ of $22.44 \mathrm{mM}$ and reaction temperature of 49.68 , respectively. The proposed mechanism of asymmetric sulfoxidations catalyzed by SPP involves three concomitant mechanisms as follows: (1) a two-electron reduction of SPP-I, (2) a single-electron transfer to SPP-I and (3) nonenzymatic reactions, including five enzymatic and two nonenzymatic reactions, which is reasonable and can express the oxidations. With $5.44 \%$ of the average relative error, a kinetic model based on the mechanisms fitting observed data very well was established, and the SPP-catalyzed reactions including both the two-electron reduction and the single-electron transfer mechanisms obey ping-pong mechanism with substrate and product inhibition, while nonenzymatic reactions follow a power law. This study has also demonstrated the feasibility of SPP as a substitute with low cost, excellent enantioselectivity and better thermal stability.
\end{abstract}

\section{Hosted file}

Biotechnology and Bioengineering.pdf available at https://authorea.com/users/364771/articles/ 485069-synthesis-of-s-omeprazole-catalyzed-by-soybean-pod-peroxidase-in-water-in-oilmicroemulsions-optimization-and-modeling 